\title{
Migration de particules dans un écoulement turbulent
}

\author{
Migration of particles inside a turbulent flow
}

\author{
H. Burnage \\ Professeur à l'Université Louis Pasteur de Strasbourg
}

L'une des propriétés essentielles de la turbulence est de réduire considérablement le temps de transfert de la quantité de mouvement de la chaleur et de la masse. Ceci est également vrai lorsque l'écoulement est diphasique et que la masse transférée est celle de particules solides ou liquides.

On se propose, dans cet exposé, de présenter une méthode d'étude de tels transferts basée sur la trajectographie des particules; on montre que cette approche, qui suscite actuellement beaucoup d'intérêt, a l'avantage de permettre de prendre en compte plus facilement d'éventuelles transformations physicochimiques des particules pendant leur migration et, le cas échant, leur action sur le fluide environnant.

One of the main properties of turbulence is to reduce strongly the rate of transfer of momentum, heat and mass. This is also true in two-phase flows where the mass transferred is that of solid or liquid particles.

We present here a method of study of these transfers which is based on the trajectography of particles. It is shown that it enables to take eventually into account the physico-chemical transformations of the particle during its motion and its action on the surrounding fluid, which is a great advantage.

\section{Introduction}

La nature lagrangienne de la diffusion de particules restreint considérablement la portée des résultats obtenus par les méthodes de calcul basés sur des modèles de type moléculaire, et, en particulier lorsque les particules qui sont transportées dans un milieu turbulent réagissent contre celui-ci. Dans le cas où la phase dispersée peut être assimilée à des particules fluides passives, des calculs basés sur l'analogie du transfert de quantité de mouvement de chaleur et de masse permettent, dans certaines configurations d'obtenir des résultats satisfaisants, mais quand celles-ci ont une taille plus grande que la micro-échelle de Kolmogorof, qu'elles opposent une inertie au fluide qui les entraine où qu'elles subissent une transformation physico-chimique, leur présence active doit être prise en compte; d'où l'avantage d'une approche qui donne accès au comportement de la particule traitée individuellement.

\section{Simulation des trajectoires de particules pas- sives}

Le principe de la trajectographie repose sur les bases suivantes : Soit une particule $P$ dont le mouvement est observé dans un reprère cartésien $\left[0, x_{i}\right]$ (avec $i=1,2,3$ ), qui se trouve à l'instant $t$ en un point $A$. En première approximation, elle atteindra le point $B$ à l'instant $t+\Delta t$ tel que :

$$
x_{i}(B)=x_{i}(A)+v_{i}(A) \cdot \Delta t
$$

où $v_{i}(A)$, vitesse de la particule en $A$ à l'instant $t$, est une fonction aléatoire devant remplir certaines conditions. On 
obtient alors la trajectoire, sous forme d'une ligne brisée, en procédant au tirage qui donne $v_{i}(A)$ que l'on multiplie par le pas de temps que l'on a choisi $\Delta t$, ce qui permet, après avoir ajouté la coordonnée issue du tirage précédant, de connaitre la nouvelle position de la particule. On dessine la trajectoire en répétant autant de fois que celà est nécessaire cette opération (figure 1).

La relation (1) présentée sous cette forme parait, d'un point algébrique, limitée au premier ordre, cependant on ne recherche pas ici une précision sur la position de la particule mais à obtenir un ensemble de positions considérées comme variables aléatoires dont les propriétés statistiques soient les mêmes que celles des positions des particules réelles. Par conséquent tout le problème de la simulation consiste à déterminer les procédés de tirage au sort de ces variables afin que l'ensemble des trajectoires et du champs des vitesses simulés satisfassent trois conditions fondamentales : 1) En tout point la distribution en l'amplitude des vecteurs vitesses fluctuants doit correspondre à celle réellement observée, 2) les fluctuations locales de vitesses doivent, en moyenne être compatibles avec la distribution locale du tenseur de Reynolds, 3) le champs des vitesses utilisé pour la simulation doit satisfaire la distribution du tenseur des corrélations spatiotemporelles ou lagrangiennes.

\section{Méthodes lagrangiennes}

Suivons une particule qui, à l'instant $t$ est animée de la vitesse $v_{i}(t)$ et à l'instant $t+\Delta t$ de la vitesse $v_{i}(t+\Delta t)$, où $v_{i}(t)$ est une fonction aléatoire et considérons la composante $\imath_{\iota}$. Soient $x$ et $y$ deux autres variables aléatoires centrées normées et statistiquement indépendantes. On peut écrire:

$$
\begin{gathered}
v_{1}(t)=a_{1} \cdot x \\
v_{1}(t+\Delta t)=a_{2} \cdot x+b_{2}, y
\end{gathered}
$$

En supposant l'homogénéité on a :

$$
\overline{v_{1}(t) v_{1}(t)}=\overline{v_{1}} \overline{(t+\Delta t), v_{1}(t+\Delta t)}=\sigma^{2}\left(v_{1}\right)
$$

En prenant le carré moyen de (2) et (3) et la moyenne de leur produit, il vient :

$$
\begin{gathered}
v_{1}(t)=\sigma\left(v_{1}\right) \cdot x \\
v_{1}(t+\Delta t)=R_{t, 1} \cdot v_{1}(t)+\left[1-R_{t, 1}^{2}\right]^{1 / 2} \cdot \sigma\left(v_{1}\right) \cdot y(5)
\end{gathered}
$$

où : $R_{t .11} 1=\overline{v_{1}(t) \cdot v_{1}(t+\Delta t)} / \sigma^{2}\left(v_{1}\right)$ est le coefficient de corrélation lagrangienne.

C'est la relation de base qui permet la simulation. En effet, l'intervalle de temps $\Delta t$ étant fixé, si une particule se trouve en $A$ à l'instant initial $(t=0)$, sa vitesse sera obtenue par (4), sa nouvelle position $B$ par (1) et la valeur de sa vitesse en $B$ par (5), puis on effectue un nouveau tirage en considérant $B$ comme la nouvelle origine spatiotemporelle du mouvement, on procède de la même façon avec $v_{2}$ et $v_{3}$, et l'on obtient, en recommençant un certain nombre de fois l'opération, les trajectoires en lignes brisées de la figure 1.

La densité de probabilité en amplitude des vitesses intervient dans le choix des distributions de $x$ et de $y$ qui, comme on l'a dit plus haut sont supposées gaussiennes pour tous les auteurs, ce qui n'est qu'une hypothèse. Les autres inconnues du problème sont $\sigma\left(v_{i}\right)$ et $R_{l}$. La forme généralement adoptée pour la fonction de corrélation lagrangienne est

$$
R_{i, 1}=\operatorname{Exp}\left[-\Delta t / T_{t}\right]
$$

où $T_{L}$ est l'échelle de temps lagrangienne. Celle-ci est liée au temps d'autocorrélation eulérien $T_{F}$ par l'hypothèse de Batchelor (1957) qui suppose que, localement, $T_{l}$ est proportionnel à $T_{E}$ et que $\sigma\left(v_{i}\right)$ est également proportionnel à $\sigma\left(u_{i}\right), u_{i}$ étant la vitesse eulérienne.

\subsection{Méthodes eulériennes-lagrangiennes}

Dans les cas où l'écoulement est complexe et la turbulence fortement inhomogène, il devient nécessaire d'imposer aux fluctuations de vitesse qu'elles soient également compatibles avec le tenseur de Reynolds local dont les élements peuvent être connus soit par des résultats d'expérience soit par le calcul (la méthode du $K-\varepsilon$ par exemple).

On procède d'une manière analogue à celle présentée plus haut mais en introduisant, dans le cas tridimensionnel, trois variables aléatoires centrées normées et statistiquement indépendantes.

Si $x, y, z$ sont ces variables, on posera :

$$
\begin{gathered}
v_{1}=a_{1} x \\
v_{2}=a_{2} x+b_{2} y \\
v_{3}=a_{3} x+b_{3} y+c_{3} z
\end{gathered}
$$

D'où l'on tire :

$$
\begin{gathered}
v_{1}=\sigma\left(v_{1}\right) x \\
v_{2}=\sigma\left(v_{2}\right)\left[x F_{1}+y F_{2}\right] \\
v_{3}=\sigma\left(v_{3}\right)\left[x G_{1}+y G_{2}+z G_{3}\right]
\end{gathered}
$$

où : $F_{1}, F_{2}, G_{1}, G_{2}$ et $G_{3}$ sont des fonctions des coefficients de corrélation eulériens au point considéré $R_{12}, R_{23}$ et $R_{31}$.

Les trajectoires sont alors obtenues en appliquant la relation (1) sous forme de lignes brisées. Cette méthode a été proposée par Ormancey (1983) et Ormancey et Martinon (1983). Les auteurs tiennent également compte de l'effet de la corrélation lagrangienne de la manière suivante :

Celle-ci étant toujours définie par la relation (6), ils font appel à une quatrième variable aléatoire $q$ uniformé-

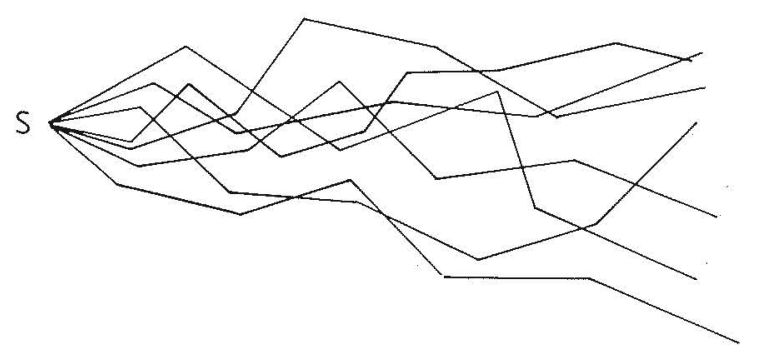

1. Simulation de trajectoires. 


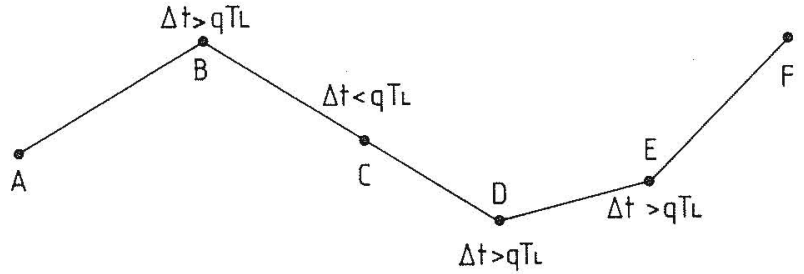

2. Prise en comple de la corrélation lagrangienne (Martinon ef Ormancey, 1983).

ment répartie dans le domaine $(0,1)$; soit $q$ une réalisation de cette variable au moment où la particule arrive en un point, si $\Delta t<q . T_{t}$, la particule conserve sa vitesse et se dirige vers le point suivant sur le même segment de droite, dans le cas contraire on effectue un nouveau tirage ( $f$ ig. 2 ), $T_{i}$ est calculé en utilisant la relation de Corrsin (1963) et est une valeur locale que l'on peut déterminer en chaque point puisque les résultats du calcul de l'écoulement moyen donnent les éléments nécessaires à son évaluation.

\section{Simulation des trajectoires de particules réactives}

Lorsque les particules dont on étudie la trajectoire ne sont plus des particules de fluide et que leur masse volumique est différente de celle du milieu environnant, leur comportement est fortement influencé par les forces dues à l'action du fluide sur elles. Elles peuvent être par ailleurs le siège de transformations physico-chimiques qui influent sur leur mouvement en modifiant, par exemple, leur taille. Quand elles sont purement inertielles et en faible concentration on admet qu'elles ne perturbent pas le milieu turbulent porteur et dans ce cas on peut se limiter à l'étude de leur dispersion en tenant compte, par des tests appropriés tels que celui proposé par Ormancey et Martinon (1983), de l'effet de croisement des particules avec les structures turbulentes en mouvement. Toutefois l'une des applications essentielles de ces méthodes est la prédiction du comportement de particules qui au cours de leur trajet subissent une transformation physico-chimique comme, par exemple, une évaporation ou une combustion. Dans ces conditions, le changement de phase subi par la particule transfère du gaz et de l'enthalpie au milieu turbulent porteur qui en sera modifié. Ceci peut être formalisé quand l'écoulement turbulent est déterminé par une algorithme de calcul basé sur les équations fondamentales en complétant chacune des ses équations par un terme source qui exprime le taux de création de masse, de quantité de mouvement et d'énergie qui résulte de la transformation physico-chimique des particules.

\section{Un exemple d'application}

On se bornera ici à comparer les résultats donnés par une simulation obtenue par la méthode d'Ormancey et Martinon (1983) aux données obtenues expérimentalement par Huilier (1983).

Les expériences ont été effectuées dans une veine d'expérience de section carrée et munie dans sa section d'entrée d'une grille de turbulence. L'injecteur était constitué par un tube cylindrique dont l'axe coïncidait avec celui de la veine et qui introduisait à vitesse isocinétique, en aval de la grille, un mélange d'air et de gouttelettes submicromiques de dioctylphtalate à faible concentration. Des mesures de pression et anémométriques par fils chauds avaient permis au préalable de vérifier que la turbulence de grille avait bien les caractéristiques classiques et de déterminer les différentes lois de décroissance et les échelles de corrélation. Les mesures de concentration ont été effectuées par des méthodes optiques basées sur la diffusion de la lumière et ont donné la distribution des concentrations de dioctylphtalate dans le champ de l'écoulement. On déduit des profils de concentration la variance du déplacement transversal des gouttelettes qui fait l'objet de la figure 3 où Rem est le nombre de Reynolds basé sur la maille de la grille.

Dans les programmes de simulation on a tenu compte de la dimension finie de l'injecteur en répartissant de façon aléatoire et uniforme le point d'émission des particules.

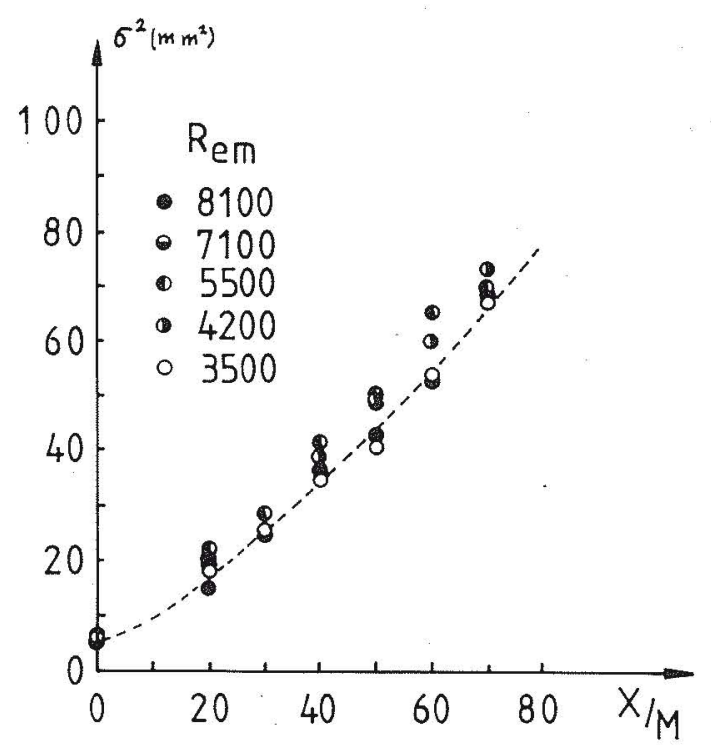

3. Application à la diffusion d'un contaminant passif. 


\section{Conclusion}

Il existe d'autres méthodes de calcul de la diffusion de particules que celles basées sur des simulations de trajectoires. Les plus récentes prennent en compte l'effet réactif des particules comme par exemple celles développées par Picart, Berlemont et Gouesbet (1986) et TsanHsing Shih et Lumley (1986) qui paraissent donner des résultats qui vérifient bien ceux de l'expérience et il ne saurait être question de présenter l'approche par simulation comme seule capable de résoudre le problème. Il faut remarquer qu'ici les difficultés sont dûes comme toujours au manque d'informations sur les statistiques eulériennes et lagrangiennes des écoulements turbulents ce qui se traduit en particulier par la sensibilité des trajectoires à l'intervalle de temps $\Delta t$ qui sépare les tirages. La plupart des auteurs mentionnent, en effet, que la précision des résultats dépend de la valeur donnée à ce paramètre sans donner de critères de choix. Par ailleurs, comme on l'a déjà souligné, la distribution en amplitude des fluctuations de vitesses est toujours supposée gaussienne ce qui est rarement le cas dans les écoulements réels mais là aussi les résultats de mesures qui permettraient de tenir compte des variations de cette fonction sont rares.
Malgré ces lacunes, l'étude de la diffusion par simulation des trajectoires des particules propose une alternative intéressante quand les particules sont réactives puisque l'on peut introduire les lois mécaniques thermodynamiques et physico-chimiques au niveau de la particule même. Elle présente également l'intérêt de tenir compte de l'influence de ces actions sur l'écoulement porteur lorsque celui-ci est calcule en introduisant des "termes sources" dans les diverses équations.

Par ailleurs, il semblerait intéressant, à l'époque où les ordinateurs deviennent de plus en plus performants, de tirer parti de leur puissance pour en faire le dispositif expérimental privilégié de nouvelles études sur la description lagrangienne de la turbulence.

\section{Adresse de l'auteur}

Monsieur H. Burnage

17, avenue du Général de Gaulle, 67000 Strasbourg.

\section{Bibliographie}

Batchelor, G.K. - Diffusion in free turbulent shear flow. J.F.M., 3, p. 67-80, 1957.

CORRSIN S. - Estimation of the relation between eulerian and lagrangian scales in large Reynolds number turbulence. $J$. Atmos. Sci., 20, p. 115-119, 1963.

Huilier, D. - Contribution à létude de la diffusion d'un aérosol dans une turbulence de grille, Thèse de $3^{\text {c }}$ cycle, U.L.P. Strasbourg, 1983.

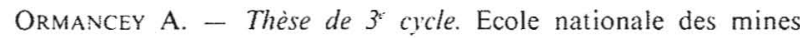
Paris, 1983.
Ormancey A., Martinon J. - Simulation numérique du comportement de particules dans un écoulement turbulent. La Recherche Aérospatiale, 5, p. 353-362, 1983.

Picart A., Berlemont A., Gouesbet G. - Modelling and predicting turbulence fields and the dispersion of discrete particles transported by turbulent flows. Int. J. Multiphase Flow; Vol. 12, № 2, 1986.

TSAN-HSING Shih, LUMLEY J.L. - Second-order modelling of particle dispersion in turbulent flow. J.F.M., 163, p. 349-363, 1986. 